\title{
aigestre
}

\section{A Política Nacional de Educação Permanente em Saúde com Foco na Segurança do Paciente}

\author{
The National Policy on Permanent Health Education with a Focus on Patient Safety
}

\author{
Fernanda Vandresen ${ }^{1}$, Maria Luiza Milani ${ }^{2}$
}

\begin{abstract}
Resumo
Introdução: A temática da pesquisa refere-se às estratégias da Política Nacional de Educação Permanente em Saúde (PNEPS) com foco na segurança do cuidado ao paciente. Destaca-se que uma importante estratégia para a gestão de risco nos serviços de saúde é a adoção de políticas públicas, como a Política Nacional de Educação Permanente em Saúde. Métodos: Esta pesquisa é de natureza aplicada e tem abordagem qualitativa com objetivo exploratório e descritivo, de caráter documental. O universo da pesquisa foi a região do Planalto Norte Catarinense. Na coleta de dados, explorou-se o Plano Regional de Educação Permanente em Saúde do Planalto Norte-Catarinense, publicações da Comissão de Integração Ensino-Serviço e documentos da Secretaria Estadual de Saúde de SC. Resultados: Foi possível identificar que a PNEPS tem reconhecida importância na região, pois por meio da implementação de uma política pública idealizada em nível nacional, considera as especificidades e necessidades regionais para traçar objetivos com foco nos profissionais e indicadores de saúde. Conclusão: Os dados evidenciaram as iniciativas que se concretizaram ou estão em consolidação sob influência de aspectos históricos, políticos e culturais de cada município, e a existência ou não de um Núcleo de Segurança do Paciente (NSP) atuante.
\end{abstract}

Palavras-Chave: Educação Permanente. Segurança do Paciente. Política Pública.

\begin{abstract}
Introduction: The research theme refers to the strategies of the National Policy of Permanent Education in Health (PNEPS) focused on the safety of patient care. I emphasize that an important strategy for risk management in health services is the adoption of public policies such as the National Policy on Continuing Education in Health. Methods: This research is applied in nature and has a qualitative approach with an exploratory and descriptive objective, of documentary nature. The research universe was the Planalto Norte Catarinense region. In the data collection, we explored the Regional Plan of Permanent Education in Health of the Planalto Norte-Catarinense, publications of the Commission of Integration Teaching-Service and documents of the State Department of Health of SC. Results: It was possible to identify that the PNEPS has recognized importance in the region, because through the implementation of an idealized public policy at the national level, it considers regional specificities and needs to set objectives with a focus on professionals and health indicators. Conclusion: The data evidenced the

1 Mestre. Universidade do Contestado - Canoinhas. Email: enf_fv@yahoo.com.br 2 Doutora. Universidade do Contestado - Canoinhas. Email: marialuiza@unc.br Correspondência: Universidade do Contestado, Programa do Mestrado em Desenvolvimento Regional - Rua Roberto Elke, 86 Centro. Canoinhas, SC - Brasil. CEP: 89460000.
\end{abstract}


initiatives that have been or are being consolidated under the influence of historical, political and cultural aspects of each municipality, and the existence or not of an active nucleus of patient safety (NPS).

Keywords: Education, Continuing. Patient Safety. Public Policy.

\section{Introdução}

A temática da pesquisa refere-se às estratégias da Política Nacional de Educação Permanente em Saúde (PNEPS) com foco na segurança do cuidado ao paciente.

No Brasil, a Política Nacional de Educação Permanente em Saúde (PNEPS) foi instituída em 2004, por meio da Portaria Ministerial №198, tendo como proposta a transformação das práticas de saúde, visando à melhoria da formação mediante uma reflexão e possibilidade de transformação da prática dos profissionais ${ }^{1}$.

Em 28 de novembro de 2017, por meio da Portaria MS nำ.194, foi instituído - Programa para o Fortalecimento das Práticas de Educação Permanente em Saúde no SUS (PRO EPS-SUS). O PRO EPS-SUS tem como principal objetivo "estimular, acompanhar e fortalecer a qualificação profissional dos trabalhadores da área da saúde para a transformação das práticas"2.

É importante salientar que a segurança do paciente está obtendo cada vez mais visibilidade, tendo recebido crescente atenção no campo dos serviços de saúde. Apesar da melhoria contínua no aprimoramento de novas técnicas, procedimentos e processos de trabalho, ainda há ocorrência de eventos adversos que atingem o paciente e comprometem a sua saúde. Sendo assim, salienta-se que receber uma assistência à saúde segura é um direito das pessoas que se submetem aos seus cuidados ${ }^{3}$.

A esse respeito, considera-se que é necessária uma contínua busca por conhecimentos técnico e científico na atuação profissional, e as práticas profissionais não devem ter embasamento empírico, mas sim acompanhar a evolução técnico-científica e a medicina baseada em evidências ${ }^{4}$.

Deste modo, torna-se um desfio conferir credibilidade e confiabilidade no processo de trabalho dos profissionais da saúde. Essas premissas apontam que se devem considerar os princípios da educação permanente para favorecer intervenções qualificadas.

A melhoria na qualidade da assistência prestada pelos profissionais de saúde, atualmente tem recebido atenção especial, não só no Brasil como no âmbito global. "A Organização Mundial de Saúde (OMS) estima que todos os anos dezenas de milhares de pessoas sofrem danos desnecessários 
causados por serviços de saúde inseguros" 5 .

$$
\text { Estudos apontados pela }
$$

Organização Mundial da Saúde (OMS) sobre a ocorrência de eventos adversos (EA) na assistência à saúde, demonstram que estes são a décima quarta maior causa de morbidade e mortalidade em todo o mundo 6 .

Estatísticas apontam que o ano de 2017 registrou 54.076 óbitos por falhas nos hospitais brasileiros. Esses indicadores estão relacionados a causas diversas e graves resultantes de infecções, complicações com acessos vasculares, erros no uso de medicações, complicações cirúrgicas e hemorragias. Dados do II Anuário da Segurança Assistencial Hospitalar no Brasil, produzido pelo Instituto de Estudos de Saúde Suplementar (IESS) e pelo Instituto de Pesquisa Fundação Educacional Lucas Machado (FELUMA), apontam que morrem por dia 148 pessoas decorrentes de erros assistenciais ${ }^{7}$.

Os impactos das ocorrências vão muito além do sentimento de insegurança do paciente, pois os prejuízos causados a este influenciam no meio familiar, proporcionando sofrimento psíquico e quebra da confiança do mesmo nos profissionais e nos serviços de saúde ${ }^{8}$.

Diante do exposto, uma importante estratégia para a gestão de risco nos serviços de saúde é a adoção de políticas públicas como a Política Nacional de Educação Permanente em Saúde.
Por isso, para além de receber assistência à saúde de qualidade como um direito inegável das pessoas, urge a primordialidade em realizar estudos, pesquisas, análises e constituir argumentos com a finalidade de conhecer os serviços para subsidiar a sensibilização dos profissionais, dos gestores e, em especial, da sociedade pelos dados encontrados, visando minimizar os erros e, por sua vez, as consequências indesejáveis relacionadas a assistência à saúde.

Diante destas questões, definiu-se como objetivo do presente estudo refletir sobre a Política Nacional de Educação Permanente em Saúde, aplicada no cenário do Planalto Norte-Catarinense com foco na segurança do cuidado ao paciente.

\section{Métodos}

A presente pesquisa é de natureza aplicada e tem como forma de abordagem qualitativa com objetivo exploratório e descritivo, de caráter documental.

No intuito de refletir acerca das ações da PNEPS no Planalto NorteCatarinense (PNC) com foco na segurança do cuidado ao paciente, optouse por uma pesquisa aplicada com abordagem qualitativa visando obter uma visão mais ampla do cenário da Educação Permanente em Saúde na região.

O universo de pesquisa foi 0 Planalto-Norte Catarinense. A região é 
formada por 13 municípios: Bela Vista do Toldo, Campo Alegre, Canoinhas, Irineópolis, Itaiópolis, Mafra, Major Vieira, Monte Castelo, Papanduva, Três Barras, Porto União, Rio Negrinho e São Bento do Sul. A população é formada por aproximadamente 366.928 habitantes $^{9}$.

A coleta de dados foi realizada mediante consulta nos Planos de Ação Regional de Educação Permanente em Saúde - PAREPS 2014/2017 e 20182021. Intencionou-se uma verificação do planejamento pactuado das ações e as ações realizadas e planejadas; publicações do Centro Integrado de Educação e Saúde (CIES) de processos avaliativos de Educação Permanente em Saúde em Santa Catarina e documentos da Secretaria Estadual de Saúde de Santa Catarina.

\section{Resultados}

\section{A Política Nacional de Educação Permanente em Saúde no Planalto Norte-Catarinense}

A Política Nacional de Educação Permanente em Saúde (PNEPS) foi implantada no Estado de Santa Catarina em 2007, e no PNC, em 22 de abril de 2008. A implementação desta política na região oportunizou a criação de espaços de discussão, por meio do CIES, e conta com a construção da Política Pública via atores sociais envolvidos na gestão, ensino, assistência e controle social. Os 13 (treze) municípios do PNC estão representados no CIES, e possuem regimento interno que designa as finalidades para efetivação da PNEPS. "Estes segmentos integram a base para a efetividade na Educação Permanente em Saúde (EPS), dentro dos municípios"10.

As reuniões do CIES para elaboração das propostas de trabalho e planejamento das ações no PNC foram pactuadas para se realizarem na última segunda-feira de cada mês. Anualmente, a agenda de trabalho é definida e pactuada pelos integrantes ${ }^{11}$.

As discussões iniciais resultaram na aprovação do Plano de Trabalho 2008/2012, elencando as prioridades regionais. O primeiro PAREPS foi aprovado em 2012, porém, algumas dificuldades foram encontradas, principalmente devido à mudança da representante do CIES. Esta era articuladora em 2013, o que acabou ocasionando a desestruturação do grupo momentaneamente. No mesmo ano, os atores envolvidos se reuniram e optaram por retomar ao planejamento e fortalecer as ações ${ }^{10}$.

As dificuldades encontradas diante da mudança de atores envolvidos induzem a uma reflexão sobre o compromisso individual de cada pessoa envolvida. Isso porque os processos de construção e discussão mostram um personalismo e uma imaturidade política na sociedade, resultando em planejamentos e projetos centrados em indivíduos, e não na coletividade. 
Outras dificuldades foram apontadas no planejamento, como "investimentos restritos em qualificação profissional, educação e saúde; a região sempre foi muito desassistida em serviços que o Estado oferece"12.

Reconhecendo as limitações, o grupo de trabalho sugeriu a criação dos Núcleos Municipais de Educação Permanente em Saúde (NMEPS), cuja intervenção destes é organizar os planos municipais de EPS que, posteriormente, seriam compartilhados em nível regional. "Atualmente, a CIES conta com nove munícipios com os NMEPS atuantes e em processo de fortalecimento e quatro municípios em fase de implantação"10.

A implantação no Núcleo Municipal de Educação Permanente em Saúde pode proporcionar discussões enriquecedoras entre os diferentes atores envolvidos na gestão e no processo de cuidado, contando também com a participação da sociedade. Se conduzido de forma atuante, reflexões de questões cotidianas conseguem reconduzir a transformações positivas nos serviços de saúde e nas condições de trabalho dos profissionais.

Os integrantes do CIES reconhecem o PAREPS como instrumento importante na orientação da política pública de saúde, pois após sua construção, este documento está em permanente revisão, visando garantir o alcance dos objetivos estabelecidos.
O PAREPS do Planalto NorteCatarinense é pautado nas necessidades regionais, e sua construção se dá por meio de oficinas com a participação dos membros do CIES dos 13 municípios, levando em consideração os indicadores de saúde. Após sua aprovação, as ações são executadas em cooperação com os atores do quadrilátero da formação (gestores, profissionais, ensino e usuários), e possíveis adequações, durante a execução do plano. Podem ser "realizados pelos órgãos de controle social e as demais esferas de governo, de forma a criar e consolidar uma política de saúde legítima e participativa"12.

A Política Nacional de Educação Permanente em Saúde tem reconhecida importância na região do Planalto NorteCatarinense. Com a implementação de uma política pública idealizada no Brasil, com ferramentas para sua descentralização e regionalização, considera-se as especificidades e necessidades regionais para, por intermédio do Plano Regional de Educação Permanente em Saúde, traçar objetivos com foco nos profissionais e indicadores de saúde.

Nesse âmbito, as ações planejadas em nível regional contribuem para minimizar as diferenças no país, e isso pode potencializar o desenvolvimento ${ }^{13}$. Porém, sobre este aspecto, alguns elementos dificultam os resultados no âmbito da saúde regional, como: capacidade limitada dos gestores para 
planejar, número insuficiente de profissionais de saúde, dificuldade dos servidores em trabalhar de forma integrada, fragilidade nas relações interestaduais, entre outros ${ }^{14}$.

A possibilidade de avaliação dos impactos e resultados das ações da Educação Permanente em Saúde em Santa Catarina (SC) iniciou mediante a criação de um instrumento, que foi elaborado após realização de oficinas de construção com participação dos integrantes do CIES do estado de SC, iniciadas em 2014. De setembro a dezembro de 2017, duas regiões de saúde aplicaram o instrumento avaliativo para analisar a viabilidade e, em oficinas estaduais realizadas em 2018, foram feitos os ajustes necessários. A matriz avaliativa será aplicada por um articulador municipal e posteriormente os resultados serão socializados. $O$ relato de experiência sobre a elaboração do instrumento avaliativo recebeu 0 reconhecimento da Organização Pan Americana da Saúde (OPAS) e do Ministério da Saúde (MS) como inovação no campo da EPS no Brasil, em julho de $2018^{15}$.

Considerando a tríade planejamento-metodologia-avaliação, prática indispensável na PNEPS, a aplicação deste instrumento avaliativo se tornou uma forma de acompanhamento e avaliação da Política Pública, tanto em nível regional quanto municipal. Reconhecendo estes dados, indicadores de desempenho poderão ser definidos e acompanhados para corrigir as fragilidades e fortalecer as potencialidades. Assim, ao obter um diagnóstico situacional, os atores envolvidos podem sentir-se provocados a refletir a respeito dessa autoavaliação e promover mudanças significativas.

Em pesquisa que avaliou a Educação Permanente (EP) nos serviços públicos, os autores apontam uma autocrítica ao processo, devido à ausência de estudo sobre as metodologias utilizadas, tampouco das avaliações realizadas após as atividades desenvolvidas. Relatam que existe implantado um instrumento de avaliação simples, com a possibilidade de 0 participante sugerir os próximos cursos, porém não há tabulação e divulgação de dados para possíveis melhorias ${ }^{16}$.

Voltando ao PNC, são apontadas como limitações para a consolidação efetiva da PNEPS no PNC: pouca participação dos gestores, resistência por parte de alguns profissionais quanto às diretrizes da Política Pública (PP), a identificação de parâmetros para os projetos e ações, a burocracia para aprovação dos projetos, necessidade de aprimorar a organização dos cursos de educação à distância ${ }^{10}$.

Dessa forma, e sob tal complexidade, é imprescindível que os profissionais de saúde envolvidos no processo de cuidar, reconheçam a necessidade de melhoria nos processos 
do seu trabalho, pois não basta ter domínio técnico, é preciso domínio político e estratégico, para que todos os envolvidos, desde os gestores aos serviços de apoio, despertem interesse em consolidar a PNEPS.

Frisa-se que, não apenas os integrantes do CIES têm responsabilidades sobre os objetivos traçados no PAREPS, mas também todos os profissionais que atuam nos serviços de saúde devem reconhecer a sua importância, pois ao serem colocados em prática resultam em mudança na formação e na prática profissional, que refletem em benefícios para a população.

Para contribuir com a efetivação dos direitos fundamentais, como a saúde, há a necessidade, por parte do Estado, de recursos apropriados para a sua operacionalização. Os recursos humanos fazem parte desses requisitos para a efetivação de políticas públicas que visem a qualidade da assistência e o bem-estar da população.

Com o envolvimento de gestores e profissionais de saúde, atuando de forma interdisciplinar, sustentada em princípios teóricos e conceituais, que sejam convergentes com as necessidades regionais, os serviços públicos podem maximizar a garantia do direito à saúde.

Cabe salientar que as práticas de educação permanente em saúde devem ser apropriadas aos aspectos culturais e regionais, de modo a contribuir para a melhoria no atendimento de saúde. Por isso, há a necessidade de considerar a relevância do Programa Nacional de Segurança do Paciente, bem como as diretrizes de sua implantação, que visam proporcionar um sistema de saúde mais seguro.

\section{Ações da Política Nacional de} Educação Permanente em Saúde e sua relação com a Segurança do Paciente no PNC

A segurança do paciente, por se tratar de um aspecto que reflete diretamente na qualidade do cuidado prestado, deveria ser considerada como pré-requisito, pois as instituições desenvolvem atividades assistenciais que são complexas, e isso exigem uma atualização constante dos conhecimentos e procedimentos técnicos ${ }^{17}$.

Incluir o tema segurança do paciente nas ações de Educação Permanente em Saúde faz parte do eixo 3 do PNSP, pois os avanços na saúde ocorrem de forma muito rápida, por isso os serviços, ao incorporar novas tecnologias ou acompanhar avanços nas pesquisas, devem incluir no seu planejamento ações de EPS para fornecer subsídios aos profissionais, objetivando um cuidado mais acurado.

A Organização Mundial da Saúde (OMS) reconhece que, além das falhas relacionadas aos processos de trabalho, as pessoas submetidas a cuidados de saúde estão sujeitas às violações das condutas de segurança. "Em todas as 
culturas, exige-se que todo profissional de saúde seja competente, ético e responsável por suas ações"18. Porém, ainda hoje se observam profissionais que não utilizam a técnica adequada para realizar a higienização das mãos, por exemplo. Erro causado pelo sistema é diferente de violação, pois, neste caso, o erro acontece quando a pessoa está tentando fazer a coisa certa. Já a violação é um erro que acontece quando o profissional descumpre alguma norma ou protocolo $^{18}$.

Nestes casos, uma cultura sistêmica de segurança não exime os profissionais de suas responsabilidades. Por conseguinte, são necessárias ferramentas capazes de conduzir situações com profissionais que assumem comportamentos clínicos não seguros. ${ }^{18}$ Nesse âmbito, ressalta-se que ações da PNEPS direcionadas à segurança no cuidado ao paciente podem contribuir significativamente para a redução da ocorrência de violações.

Conforme indicadores do PAREPS 2014/2017, a proporção de execução das ações de Educação Permanente em Saúde planejadas e executadas no Planalto Norte-Catarinense é de $83,33 \%$ 11.

O PAREPS também menciona oportunidades de uso de ferramentas gratuitas, como a Universidade Aberta do SUS (UNA-SUS) e o TeleSaúde que, por meio da educação permanente a distância, oportunizam o aprimoramento

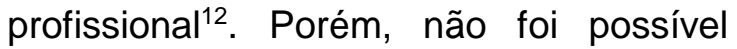
mensurar os dados nesta modalidade, devido a ausência de registros e divulgação do alcance dos cursos oferecidos. Aqui, evidencia-se 0 problema do limite das informações, precarizando a democratização do conhecimento e, por extensão, a qualificação dos processos.

Ao considerar os fundamentos do Programa Nacional de Segurança do Paciente, no PAREPS 2018/2021, algumas ações contemplam as metas de segurança do paciente. Veja o quadro 1.

Quadro 1: Propostas do PAREPS 2018/2021

\begin{tabular}{|l|l|}
\hline $\begin{array}{l}\text { METAS } \\
\text { INTERNACIONAIS }\end{array}$ & $\begin{array}{l}\text { TEMAS DE } \\
\text { CAPACITAÇÃo }\end{array}$ \\
\hline $\begin{array}{l}\text { 1- Identificar } \\
\text { corretamente o } \\
\text { paciente }\end{array}$ & - \\
\hline $\begin{array}{l}\text { 2- Melhorar a } \\
\text { comunicação entre } \\
\text { profissionais de } \\
\text { saúde }\end{array}$ & $\begin{array}{l}\text { Oficinas de } \\
\text { Acolhimento - da } \\
\text { teoria à prática } \\
\text { A interação no } \\
\text { Processo de } \\
\text { Trabalho em Saúde } \\
\text { Capacitação sobre } \\
\text { saúde vocal e } \\
\text { comunicação }\end{array}$ \\
\hline $\begin{array}{l}\text { 3- Melhorar a } \\
\text { segurança na } \\
\text { prescrição, no uso e } \\
\text { na administração de } \\
\text { medicamentos }\end{array}$ & $\begin{array}{l}\text { Uso correto de } \\
\text { Medicamentos }\end{array}$ \\
\hline $\begin{array}{l}\text { 4- Assegurar a } \\
\text { cirurgia em local de } \\
\text { intervenção, } \\
\text { procedimento e } \\
\text { paciente corretos }\end{array}$ & \\
\hline $\begin{array}{l}\text { 5- Higienizar as } \\
\text { mãos para evitar } \\
\text { infecções }\end{array}$ & \\
\hline $\begin{array}{l}\text { 6- Reduzir o risco de } \\
\text { quedas e úlceras } \\
\text { por pressão }\end{array}$ & $\begin{array}{l}\text { Capacitação dos } \\
\text { profissionais sobre } \\
\text { manejo do paciente } \\
\text { domiciliar }\end{array}$ \\
\hline Fonte: Dados da Pesquisa, \\
\hline
\end{tabular}

Fonte: Dados da Pesquisa, 2019. 
Conforme descrito no PAREPS, as ações de educação permanente foram planejadas pelos representantes do quadrilátero (ensino, gestão setorial, práticas de atenção, controle social) levando em consideração indicadores de saúde regionais.

No quadro 1, a Meta 2 - para melhorar a comunicação entre profissionais de saúde foi trabalhada com oficinas de acolhimento, abordagem sobre a interação no processo de trabalho em saúde e capacitação sobre saúde vocal e comunicação. A Meta 3, que visa melhorar a segurança na prescrição, no uso e na administração de medicamentos, foi contemplada com atividade educativa sobre o uso correto de medicamentos. E a Meta 6 - que objetiva reduzir o risco de quedas e úlceras (lesão) por pressão, foi contemplada com capacitação dos profissionais sobre manejo do paciente domiciliar. Ainda existe uma lacuna no trabalho da SP, pela ausência de propostas que abordem a Meta 1 - para identificar corretamente o paciente, a Meta 4 - para assegurar procedimentos cirúrgicos seguros e a Meta 5 - que objetiva evitar infecções.

No PAREPS 2018/2021, as propostas de capacitação também contemplam outras temáticas relacionadas a segurança no cuidado ao paciente: Implantação do Núcleo de Segurança do Paciente, Sistema de Notificações de Vigilância Sanitária
(NOTIVISA) e apoio e cuidado à gestante $^{12}$.

O apoio e cuidado à gestante faz parte de recentes abordagens nacionais da SP, considerando a complexidade envolvida na assistência materna e neonatal e o potencial de EAs a que estão sujeitos. Afinal, há no Brasil cerca de 3 milhões de nascimentos ao ano, ou seja, cerca de 6 milhões de pacientes envolvidos no processo de nascimento ${ }^{19}$.

O Programa Nacional de Segurança do Paciente (PNSP) foi instituído em 2013 e cerca de quatro anos após a determinação da obrigatoriedade da implantação do NSP, sua importância foi reconhecida e a temática incluída como proposta de estratégia da PNEPS no PNC. Até o momento, não houve divulgação de sua efetivação.

Mesmo reconhecendo que alguns aspectos do PNSP estão incluídos no PAREPS 2018/2021, a realidade reflete que o planejamento deve contemplar ações articuladas, que possam, de forma integrada, atender as 6 Metas Internacionais de segurança do paciente, bem como, a sensibilização para uma cultura de segurança.

Com a implantação dos protocolos e manutenção das metas internacionais em prol da Segurança do Paciente, as ações contribuem para um modelo de assistência bem-sucedido, o que implica promoção da saúde e prevenção de consequências desagradáveis, preveníveis. 
Salienta-se que mesmo o PNSP considerando obrigatória a implantação de estratégias como os protocolos de segurança do paciente, ainda há uma lacuna entre teoria e prática. Por isso, estratégias devem ser associadas ao programa para que suas ações sejam concretizadas e, neste caso, uma vigilância mais rigorosa possa impactar significativamente no cumprimento da legislação.

Neste caso, a PNEPS se torna uma estratégia que proporciona reflexão do cenário vivenciado pelo profissional de saúde e, deste modo, a aprendizagem significativa ajuda a reconhecer a importância do cuidado seguro e passa a ver mais sentido no que faz.

Nos hospitais, a implantação das metas está ocorrendo gradativamente, sendo que $80 \%$ já têm implantada a Meta 1 de Identificação do Paciente; 60\% implantaram a Meta 3 para Melhorar a segurança na prescrição, no uso e na administração de medicamentos; 54\% têm implantada a Meta 6 que visa a redução de risco de quedas e úlceras por pressão; 47\% afirmam que a Meta 4 para Cirurgia Segura está implantada; 47\% aderiram à Meta 2 para uma melhor comunicação entre os profissionais de saúde e $40 \%$ apenas com a Meta 5 , que mobiliza para a adequada higienização das mãos para prevenir infecções ${ }^{20}$.

\section{Conclusão}

Ao refletir sobre a temática, o presente estudo buscou caracterizar as ações da Política Nacional de Educação Permanente em Saúde, as quais estão direcionadas a segurança do cuidado ao paciente no cenário do Planalto NorteCatarinense.

Acompanhar a implementação de Políticas Públicas como a PNEPS, requer o estabelecimento de critérios, que a publicação desta, em nível federal, não é garantia de aplicação de suas diretrizes, pois os atores envolvidos influenciam diretamente de acordo com o grau de prioridade que estabelecem para 0 assunto.

Mas, para que as Políticas Públicas possam ser efetivadas, devem ser mediadas por profissionais comprometidos com seus princípios e diretrizes, e assim, não apenas pelo governo, que é o principal responsável. Os profissionais são executores que contribuem para a melhoria da assistência à saúde da população assistida.

Para isto, há de se considerar a interdisciplinaridade, para que, tanto profissionais de saúde como gestores, atuem de forma integrada, guiados por princípios convergentes, reconhecendo a saúde como direito fundamental, e para que a intervenção das normas regulamentadoras e dos princípios do Sistema Único de Saúde, contribuam com o cuidado seguro. 
Foi possível identificar que a PNEPS tem reconhecida importância na região, pois, por meio da implementação de uma política pública idealizada em nível nacional, é imprescindível considerar as especificidades e necessidades regionais para traçar objetivos com foco nos profissionais e indicadores de saúde.

São descritas diferentes estratégias adotadas pelos serviços de saúde dos municípios da região do PNC que, por meio da PNEPS, contribuem para a implantação das ações do PNSP. Os dados evidenciaram as iniciativas que se concretizaram ou estão em consolidação sob influência de aspectos históricos, políticos e culturais de cada município e a existência ou não de um NSP atuante.

Ações concretas que possam proteger o paciente quanto aos riscos relacionados a assistência à saúde precisam ser efetivadas, e por intervenção das políticas públicas, como a PNEPS contribuir para que, na ocorrência das causas indesejáveis, o foco não seja o profissional de saúde, mas sim, os fatores contribuintes para o desfecho inconveniente.

Este estudo verificou que a PNEPS se consolida como uma estratégia que, diante da vivência da aprendizagem significativa, pode contribuir para a melhoria dos processos de trabalho dos profissionais de saúde e, por decorrência, contribui para o desenvolvimento regional. Porém, ao refletir sobre o PNP e as estratégias que vão ao encontro dos eixos e protocolos, pode-se considerar que ainda há muito que avançar para incorporar as recomendações, normas, portarias e leis aos serviços de saúde no Brasil.

\section{Agradecimentos}

Ao apoio financeiro para a realização desta pesquisa como Bolsista do Programa de Bolsas Universitárias de Santa Catarina, Fundo de Apoio à Manutenção e ao Desenvolvimento da Educação Superior - UNIEDU/FUMDES, da Secretaria de Educação do Estado de Santa Catarina.

Agradeço a todos aqueles que, com paciência e generosidade, compreenderam que cursar um Programa de Mestrado requer dedicação e que nem sempre pude me fazer presente da forma como gostaria, na vida de entes queridos.

\section{Referências}

1. Ministério da Saúde (BR). Portaria n.198. Institui a Política Nacional de Educação Permanente em Saúde como estratégia do Sistema Único de Saúde para a formação e o desenvolvimento de trabalhadores para o setor e dá outras providências. Brasília, DF. 2004.

2. Ministério da Saúde (BR). Programa para o Fortalecimento das Práticas de Educação Permanente em Saúde no SUS - PRO EPS SUS. Manual Técnico. Brasília, 2018.

\section{Agência Nacional de Vigilância} Sanitária (BR). Assistência Segura: uma reflexão teórica aplicada à prática. Brasília, 2013. 
4. Kalsing RMK. A percepção da equipe de enfermagem frente à notificação de eventos adversos. Trabalho de Conclusão de Curso. Lajeado, 2012.

\section{Agência Nacional de Vigilância} Sanitária (BR). Plano Integrado para a Gestão Sanitária da Segurança do Paciente em Serviços de Saúde: Monitoramento e Investigação de Eventos Adversos e Avaliação de Práticas de Segurança do Paciente. Brasília, 2015.

6. World Health Organization. 10 facts on patient safety. March, 2018. [Acesso em 10 mai 2018] Disponível em < http://www.who.int/features/factfiles/ patient_safety/en/>.

7. Couto RC et al. II Anuário da Segurança Assistencial Hospitalar no Brasil. Universidade Federal de Minas Gerais, Belo Horizonte, 2018.

\section{Slawomirski L, Auraaen A, Klazinga N.} The economics of patient safety: Strengthening a value-base approach to reducing patient harm at national level, OECD, mar. 2017. [Acesso em 06 out 2017] Disponível em: <https://www.oecdilibrary.org/social-issues-migrationhealth/the-economics-of-patientsafety_ 5a9858cd-en>..

\section{Instituto Brasileiro de Geografia e} Estatística. Mapas. Disponível em: https://mapas.ibge.gov.br/fisicos/estaduais , atualização 2013.

10. Vendruscolo $\mathrm{C}$ et al. Frutos dos movimentos de educação permanente em saúde de Santa Catarina: caminhos e oportunidades. Porto Alegre: UNIDA, 2018.

11. Secretaria do Estado da Saúde (SC). Plano de Ação Regional de Educação Permanente em Saúde- PAREPS. Região de Saúde do Planalto Norte. 2014/2017. Florianópolis, 2014.

12. Secretaria do Estado da Saúde (SC). Plano de Ação Regional de Educação Permanente em Saúde- PAREPS. Região de Saúde do Planalto Norte. 2018/2021. Florianópolis, 2017.

13. Gadelha CAG, Costa L. A Saúde e territorialização na perspectiva do desenvolvimento. Revista Ciência \& Saúde Coletiva. Rio de Janeiro, 2011.

14. Nuske $M A$ et al. A saúde e sua relação com o desenvolvimento: um olhar crítico acerca da contribuição da saúde nas múltiplas escalas do desenvolvimento regional. In: VIII Seminário Internacional sobre desenvolvimento regional. Santa Cruz do Sul, 13 a 15 de setembro de 2017.

15. Secretaria do estado da Saúde (SC). Processos Avaliativos de Educação Permanente em Saúde Em Santa Catarina: uma roda que nunca parou de girar. Florianópolis, 2018. [Acesso em 14 nov 2018] Disponível em:

https://apsredes.org/processosavaliativos-de-eps-em-sta-catarina-umaroda-que-nunca-parou-de-girar/

16. Lino MM et al. Educação Permanente dos serviços públicos de saúde de Florianópolis, Santa Catarina. Trab. Educ. Saúde. v.7, n.1. Rio de Janeiro, mar/jun 2009.

17. Tondo JCA. Clima de segurança: percepção dos profissionais de enfermagem em um hospital de ensino. Unicamp, Campinas, 2015. Dissertação (Mestrado em Ciências da Saúde) Programa de Pós-Graduação em Enfermagem da Faculdade de Enfermagem na Universidade Estadual de Campinas, Campinas, 2015.

18. World Health Organization. Guia curricular de segurança do paciente da Organização Mundial da Saúde: edição multiprofissional. Tradução: Pontifícia Universidade Católica do Rio de JaneiroPUC-Rio, Rio de Janeiro: Autografia, 2016.

19. Ministério da Saúde (BR). Política de educação e desenvolvimento para o SUS: caminhos para a educação permanente em saúde: polos de educação 
permanente em saúde. Brasília, DF.

2004b.

20. Zanluca $\mathrm{CH}$. Atuação dos

profissionais de saúde frente ao Núcleo

de Segurança do Paciente nas

instituições do Planalto Norte-

Catarinense. Mafra: UnC, 2016. Trabalho

de Conclusão de Curso - Graduação de

Enfermagem, Universidade do

Contestado, Mafra, 2016.

RE. SAÚD. DIGI. TEC. EDU., Fortaleza, CE, v. 4, n. 1, p.03-16, jan./ago. 2019. 


\section{Como citar este artigo}

Vandresen F, Milani ML. A Política Nacional de Educação Permanente em Saúde com Foco na Segurança do Paciente. Revista de Saúde Digital e Tecnologias Educacionais. [online], volume 4, n. 1. Editor responsável: Luiz Roberto de Oliveira. Fortaleza, mês e ano, p. 03-16. Disponível em: http://periodicos.ufc.br/resdite/index. Acesso em "dia/mês/ano".

Data de recebimento do artigo: 16/06/2019

Data de aprovação do artigo: 29/07/2019 